\title{
La eliminación del libro de texto y el uso del portafolio en la asignatura de música en ESO: una experiencia para incrementar la calidad del aprendizaje y la motivación del alumnado
}

Removal of Textbook and Use of Portfolio in the Music Subject in Secondary Education: An Experience to Increase the Quality of Learning and Student Motivation

\author{
Katia Sanz Chouquet ${ }^{1}$ \\ ksanz1@alumno.uned.es \\ Universidad Nacional de Educación a Distancia, UNED, España
}

\section{Resumen:}

Se abordó el presente estudio con el objetivo de desarrollar estrategias que incrementaran la calidad de los aprendizajes y la evaluación, además de la motivación de los alumnos en la materia de Música en Educación Secundaria Obligatoria. Para ello se diseñó una experiencia en la que se eliminó el libro de texto y se utilizó el portafolio a lo largo del curso académico 2013-2014, complementando dicho uso con trabajos cooperativos, autoevaluaciones y co-evaluaciones. Se utilizó un diseño de dos grupos para comparar los

\begin{abstract}
:
The present study was conducted to develop strategies that would increase the quality of learning and assessment, and also student motivation in the subject of Music in Secondary Education. In order to do so, an experience was designed in which, instead of using the text book, the lessons throughout the academic year 2013-2014 where mediated through a portfolio. This was complemented with cooperative work, self-assessments and co-evaluations. Two-group design was used to compare the results in terms of satisfaction with the
\end{abstract}

1 Dirección para correspondencia (correspondence address):

Katia Sanz Chouquet. Dpto. de Didáctica. C/ Cantera 5, 2º A. 28260 Galapagar, Madrid (España) 
La eliminación del libro de texto y el uso del portafolio en la asignatura de música en ESO: una experiencia para incrementar la calidad del aprendizaje y la motivación del alumnado

Katia Sanz Chouquet

resultados en cuanto a satisfacción con la asignatura y medias académicas. Se completó la investigación con los datos relativos a la evaluación del clima de aula por parte del alumnado.

Los resultados muestran un aumento significativo del interés del alumnado por la materia y opiniones favorables acerca de la mejora personal en procesos de trabajo en equipo entre otros, la capacidad de apreciar estilos nuevos o la capacidad de síntesis.

\section{Palabras clave:}

Portafolio; música; trabajo colaborativo; autoevaluación; motivación; educación secundaria course and academic results. The study also reveals data on the students' assessments of classroom atmosphere. The results show a significant increase in student interest in music/the subject and favorable opinions about, among other aspects, personal improvement in team-work processes, the ability to appreciate new styles or the ability to summarize.

\section{Key words:}

Portfolio; music; cooperative work; selfassessment; motivation; secondary education.

\section{Resumé:}

La présente étude a été abordée dans le but de développer des stratégies qui permettront d'accroître la qualité de l'apprentissage et de l'évaluation et aussi de la motivation des élèves dans le domaine de la musique dans I'enseignement secondaire. Pour cela, nous sommes partis d'un expérience dans laquelle le manuel a été éliminé et on a utilisé le portefeuille tout au long de I'année scolaire 2013-2014, en complémentant cette utilisation avec le travail coopératif, I'auto-évaluation et la co-évaluations. Pour comparer les résultats en lien avec la satisfaction quant à la matière et les moyennes académiques, on a utilisé un plan de deux groupes. La recherche a été complétée par des données sur l'évaluation du climat en classe par les élèves. Les résultats montrent une augmentation significative de l'intérêt des élèves et des opinions favorables sur les processus d'amélioration personnelle dans le travail d'équipe entre autres, la capacité d'apprécier de nouveaux styles ou la capacité de synthèse.

\section{Mots clés:}

Portefeuille; musique; travail coopératif; motivation; éducation secondaire.

Fecha de recepción: 23-04-2016

Fecha de aceptación: 19-10-2016

\section{Introducción}

Hoy en día parece haber una necesidad acuciante de renovar la enseñanza derivada de los cambios tecnológicos que estamos viviendo. Capacitar a los alumnos para el mañana, para trabajos que quizás hoy ni siquiera existen, es una tarea que requiere grandes dosis de vocación, creatividad y visión de futuro. En esta vorágine de redes sociales, uso de recursos informáticos en el aula, pizarras digitales, proyectores etc. nos planteamos el uso de una herramienta que a priori no está relacionada 
con las nuevas tecnologías (aunque puede estarlo en su diseño y ejecución) pero que creemos otorga al alumno una perspectiva distinta acerca de su aprendizaje: nos estamos refiriendo al portafolio. La experiencia nos muestra cada vez con más frecuencia a alumnos desmotivados, sin ilusión por aprender y con intereses muy restringidos culturalmente hablando. Creemos que el cambio educativo debe venir no sólo de una renovación en la forma (mejores medios, uso de nuevas tecnologías) sino en el fondo, donde situamos como eje central el proceso de reflexión que hace del alumno el protagonista de su aprendizaje.

\section{Objetivos de la investigación}

El objetivo principal de la investigación es el de mejorar los procesos de aprendizaje del alumnado y su implicación en el mismo a través del uso del portafolio. De este planteamiento genérico podemos especificar objetivos más concretos que creemos, trascienden aquellos de la propia materia y proporcionan una mejora en competencias básicas en el alumno, aplicables como tal a otras materias y aprendizajes, a saber:

a) Proporcionar al alumno pautas de organización de su material de estudio.

b) Fomentar la reflexión acerca de los logros y las dificultades en su aprendizaje.

c) Crear "focos" de interés hacia la asignatura que nacen de la necesidad de tomar decisiones a la hora de abordar cuestiones prácticas.

d) Hacer consciente al alumno de lo que significa un proceso de mejora, tomando consciencia de sí mismo como protagonista de dicho proceso.

e) Estimular el pensamiento crítico.

f) Adquirir habilidades de búsqueda y selección de información.

g) Transformar contenidos que en un principio son ajenos al contexto del alumno y a sus inquietudes en aprendizajes significativos que amplíen el horizonte estético del alumno. 
La eliminación del libro de texto y el uso del portafolio en la asignatura de música en ESO: una experiencia para incrementar la calidad del aprendizaje y la motivación del alumnado

Katia Sanz Chouquet

\section{Consideraciones sobre el portafolio}

\section{Concepto}

La definición tradicional de portafolio incide en el aspecto de colección de trabajos o selección de aquellas tareas más relevantes que el alumno escoge como evidencia de su aprendizaje. Como indica Barragán (2005), responde al concepto "carpeta de aprendizajes" en castellano. El portafolio se comenzó a utilizar a partir de los años 1970 en EEUU. y está ligado al mundo del arte, arquitectura o diseño, cuyo concepto de portafolio se dirige a la recopilación de aquellos trabajos más significativos. Surge así como instrumento para demostrar determinadas competencias en el terreno profesional.

Shackelford (1996) define el portafolio del estudiante como una colección de materiales capaz de comunicar los intereses, progresos y habilidades de los estudiantes en un área concreta. Contrariamente a las formas tradicionales de evaluación, centradas en habilidades aisladas, los portafolios se muestran eficientes para mostrar el aprendizaje del estudiante en el tiempo.

El concepto de portafolio que nos interesa en el presente trabajo es el que hace referencia al proceso de aprendizaje. "Cuando los estudiantes realizan portafolios aprenden a evaluar su propio progreso como estudiantes y los profesores adquieren nuevos puntos de vista sobre sus logros docentes" (Wolf, D.P.1989, p. 35). Se ha puesto a menudo en relación el desarrollo creativo que genera una novela o un cuadro y sus correspondientes notas, bocetos, esbozos etc. que conlleva, con la idea de portafolio como elemento documentador del propio proceso de aprendizaje. Según el autor citado, el portafolio ofrece un retrato del desarrollo del que se puede aprender incluso después del momento aislado de la evaluación.

Probablemente la definición más completa y matizada de portafolio sea la ofrecida por Klenowski (2005):

"colección de trabajos que incluyen los logros individuales, tales como resultados de tareas genuinas, la evaluación del proceso, test convencionales o muestras de trabajo; documenta los logros alcanzados a lo largo del tiempo. Generalmente el individuo elige el tipo de trabajo que le sirva mejor para expresar su éxito así como para demostrar su aprendizaje respecto a un objetivo 
La eliminación del libro de texto y el uso del portafolio en la asignatura de música en ESO: una experiencia para incrementar la calidad del aprendizaje y la motivación del alumnado

Katia Sanz Chouquet

particular como podría ser la certificación o evaluación tanto formativa como sumativa. [...] El desarrollo del portafolio implica por tanto documentar, no solo los logros conseguidos sino también las autoevaluaciones, las estrategias aplicadas y el análisis sobre las experiencias de aprendizaje, por lo que es mucho más que una simple colección de las tareas"(p.39)

El portafolio nos permite documentar los aprendizajes y tomar decisiones tras su análisis, partiendo de una concepción constructivista, donde dicho aprendizaje es un proceso activo y orientado al logro de metas. El docente no es un simple transmisor de saberes, sino un mediador o guía entre el conocimiento y el estudiante. Éste, por su parte, debe asumir un rol activo y responsable en la construcción de su propio aprendizaje. Para ello hemos de proporcionarle experiencias con significado a partir de sus experiencias y la interacción con el entorno. Tharp y Gallimore (en Klenowski, 2005, p. 151) se refieren a una visión de un aprendizaje guiado donde el profesor asiste al alumno en dicha construcción de significados y donde la resolución de problemas tiene un papel primordial.

\section{Utilización del portafolio en el ámbito de la educación}

Un trabajo de portafolio puede cumplir todos los propósitos evaluativos que ofrecemos a continuación:

- Uso para la evaluación sumativa, donde se suele utilizar en conjunto con otras pruebas.

- Uso como base para certificación de competencias y selección de candidatos, sobre todo en el contexto de formación de profesorado.

- Uso con fines valorativos y de promoción, donde aparecen pruebas representativas del trabajo que muestran la competencia en relación con unos criterios establecidos y son recogidas por los evaluadores.

- Uso como apoyo para la enseñanza y el aprendizaje, donde predomina el papel formativo de la evaluación y se documenta el proceso de desarrollo de habilidades clave.

- Uso en el desarrollo profesional, donde los profesores reflexionan y valoran sus propias prácticas de enseñanza. Se desarrolla de manera colaborativa. 
La eliminación del libro de texto y el uso del portafolio en la asignatura de música en ESO: una experiencia para incrementar la calidad del aprendizaje y la motivación del alumnado

Katia Sanz Chouquet

Nuestra propuesta se centra en el portafolio como herramienta formativa, documentando el proceso de aprendizaje. Este uso está cada vez más extendido en la práctica educativa, no solo como un apreciado recurso en el ámbito del diagnóstico y la orientación (Corominas, 2000), sino como una forma de facilitar información sobre los procesos de aprendizaje. Configura así una metodología que desarrolla habilidades complejas (Barberà, 2005). La evaluación se modifica, dando lugar a procesos diferentes a los generados por la evaluación tradicional mediante exámenes (Gallardo Gil \& Sierra Nieto, 2015).

La educación musical será más relevante, en lo que respecta a la formación integral del alumnado, en la medida en que esté presente un enfoque más creativo y no tan conceptual como se ha venido haciendo hasta ahora en secundaria. Creemos que el portafolio, entendido como carpeta de aprendizaje, donde aparecen no solo los trabajos ejemplares, sino los errores, contribuye enormemente a este proceso (Alsina, 2008).

\section{Tipología del portafolio}

Existen en la literatura sobre el tema diversas tipologías (Quintana, 1996 o Shackelford, 1996). Como resumen, podríamos decir que encontramos dos grandes líneas en lo que respecta a la clasificación de portafolios:

- Portafolios de proceso, donde se documenta el aprendizaje. Dichos portafolios son mejorados de manera continua por la evaluación formativa.

- Portafolios de producto: relacionados con los de presentación o vitrina, especialmente bien acabados, cuya evaluación puede ser sumativa.

Pérez Juste (2012) establece una distinción que incide en la profundidad y objeto del portafolio, distinguiendo entre portafolios de primer nivel, como tarea acumulativa de trabajos y portafolios de segundo nivel, como tarea de síntesis, especialmente adecuado para evidenciar el dominio de competencias de cara por ejemplo a un Practicum.

Dentro de la tipología del portafolio y atendiendo a su soporte, en los últimos años se ha desarrollado el e-portfolio o portafolio electrónico, que utilizando la web y las herramientas informáticas comparte y extiende las ventajas del portafolios clásico (Rodríguez Illera et al., 2013), fundamentalmente por: 
La eliminación del libro de texto y el uso del portafolio en la asignatura de música en

ESO: una experiencia para incrementar la calidad del aprendizaje y la motivación del alumnado

Katia Sanz Chouquet

- Su multimedialidad, o posibilidad de incluir diferentes tipos de archivos (vídeos, audios, imágenes etc.).

- Su capacidad para ser compartido en tiempo real, online, con otros estudiantes y profesores.

- La posibilidad de retroalimentación de los trabajos a través de los comentarios insertados.

Este tipo de portafolio es cada vez más demandado incluso desde los ámbitos laborales, tanto por su capacidad para mostrar las habilidades en entornos informáticos del aspirante ayudando a la alfabetización digital (Barrett, 2005), como por la posibilidad de reflejar procesos de aprendizaje (Rhodes, 2010).

A ello contribuyen las demandas del Espacio Europeo de Educación Superior, que ha impulsado el uso de este soporte de portafolio en el ámbito universitario, "contagiando" después su uso en otros ámbitos. Encontramos una interesante síntesis en Barberà, et al. (2009).

\section{Portafolio y evaluación}

Una de las ventajas del uso del portafolio (Estebaranz y Mingorance, 1995) es su vinculación con la evaluación, en el sentido de que proporciona desde el inicio un criterio que guía la ejecución y el análisis. La evaluación deberá, ayudándose de continuos feedbacks, determinar el grado en que se han logrado los propósitos educativos. La cultura del portafolio concibe una evaluación que reúne las siguientes condiciones:

1. Está enfocada a conocimientos y destrezas que son importantes para el área en cuestión.

2. Contribuye a la enseñanza y al aprendizaje, por lo cual es necesario evaluar de manera periódica y no únicamente al final del trimestre o el año.

3. Sirve a un proceso evaluador que informa de oportunidades de aprendizaje de los alumnos y de sus éxitos y fracasos desde un punto de vista procesual.

La evaluación alternativa comprende múltiples métodos para demostrar el aprendizaje y hace la evaluación más justa, reduciendo la dependencia de un solo examen final. Por su capacidad para mostrar habilidades que las pruebas estandarizadas dejan de lado, ha surgido la 
La eliminación del libro de texto y el uso del portafolio en la asignatura de música en ESO: una experiencia para incrementar la calidad del aprendizaje y la motivación del alumnado

Katia Sanz Chouquet

necesidad de elaborar rúbricas que reflejen criterios comunes a la hora de evaluar las producciones de los alumnos, sobre todo en el ámbito universitario (Rhodes, 2010). La necesidad de unificar criterios evaluativos se refleja desde hace tiempo en la bibliografía, (Calfee y Perfumo, 1993), constatando la dificultad de conectar la cultura de la evaluación externa sumativa con el carácter más cualitativo del portafolio.

\section{Fases en la elaboración del portafolio}

Todo trabajo que documente un proceso, como es el caso del portafolio, debe tener unas fases para su realización, que garanticen su ejecución en el tiempo previsto y la adecuada selección y pertinencia de la información. Las propias fases de realización pueden servir a la vez de modelo para la estructura del portafolio. Barberà (2005) sugiere que el portafolio debería organizarse de acuerdo a las siguientes fases:

- Recogida de evidencias, donde se hace acopio de todo el material susceptible de ser incluido en el mismo.

- Selección de aquellas actividades que mejor evidencian el proceso de aprendizaje o que mayor nivel de calidad poseen.

- Reflexión acerca de dichas evidencias.

- Publicación del portafolio con una estructura ordenada y comprensible.

\section{Eliminación del libro de texto}

El panorama actual muestra un incremento paulatino del empleo de medios digitales de aprendizaje y en todo caso, alternativos a recursos como el libro de texto, más centrados estos en la enseñanza que en el aprendizaje (Lorenzo-Quiles et al. 2015, Crawford, 2014). En este contexto de renovación metodológica, vemos que aún hay ciertas reticencias a la eliminación del libro de texto como soporte único (Monereo, 2010), a pesar de los estudios que confirman la eficacia de programas que utilizan otros medios alternativos (Alsina \& Godoy, 2009). El presente estudio ha tenido como uno de sus planteamientos de base la eliminación del libro de texto, dado que creemos que este tipo de intervenciones eliminan dicho libro como "refugio" del docente reticente al cambio (Monereo, 2010) y suponen una alternativa innovadora. 


\section{Experiencia didáctica}

\section{Participantes}

Por las características del presente estudio la muestra está formada por individuos pertenecientes a grupos ya formados: los grupos de clase.

El lugar donde se ha llevado a cabo el estudio es un centro de enseñanza infantil, primaria, secundaria y bachillerato de la localidad de Pozuelo de Alarcón, ubicado en la zona oeste de la Comunidad Autónoma de Madrid. El municipio cuenta con unos 43,000 habitantes, aunque los alumnos que acuden al centro pertenecen también a Majadahonda e incluso Madrid capital. Es un centro privado, católico y bilingüe, en el que se imparte la enseñanza diferenciada (niños separados de niñas desde los 6 a los 17 años de edad). En $2^{\circ}$ de bachillerato algunas de las materias (principalmente las optativas) son mixtas.

El entorno del centro es una zona de alto poder adquisitivo, donde se encuentran bastantes centros de enseñanza privados que han apostado por el bilingüismo desde hace años. Ofrecen la mayoría de ellos, como incentivo para las familias, unas buenas instalaciones, educación en valores, atención personalizada y buenos resultados en las pruebas de acceso a selectividad.

La muestra total es de 197 alumnos pertenecientes a los cursos de $2^{\circ}$ y $3^{\circ}$ de ESO.

\section{Muestra 1.}

Para la cumplimentación de los cuestionarios sobre motivación e interés y una comparativa en resultados académicos se eligió al total de197 alumnos de los cursos de $2^{\circ}$ y $3^{\circ}$ de ESO.

Muestra 2

Una muestra de 111 alumnos es la que rellena el micro-instrumento de clima de aula. Son aquellos alumnos que constituyen el grupo experimental.

\section{Hipótesis}

Dado el campo en el que nos movemos, nuestras hipótesis tienen el carácter de "probable" y tratan de proporcionar un marco en el que situar 
La eliminación del libro de texto y el uso del portafolio en la asignatura de música en ESO: una experiencia para incrementar la calidad del aprendizaje y la motivación del alumnado

Katia Sanz Chouquet

diseños más experimentales en un futuro, gracias a un mayor aislamiento de las variables.

Creemos que la revisión de las fuentes ha fundamentado la consideración del portafolio como herramienta de gran valor educativo con todas sus implicaciones.

Considerando el planteamiento y contexto de nuestra investigación y dado que los cambios metodológicos introducidos van encaminados a la mejora, nuestra hipótesis de partida está directamente relacionada con estos cambios.

H.1. El grado de interés y satisfacción por la materia aumenta cuando se produce una reflexión continua sobre los procesos propios de aprendizaje, estableciendo objetivos y detectando los logros y dificultades a través de una herramienta como el portafolio.

H.2. El grado de interés y satisfacción por la materia aumenta por lo general cuando la metodología no se centra en el libro de texto como material único sino que se utilizan actividades diversas y proyectos colaborativos.

H.3. El uso del portafolio y de actividades de aprendizaje significativas aumenta la capacidad del alumno en cuanto a síntesis, trabajo en equipo y manejo de las TIC.

H.4. La metodología que integra un enfoque práctico y colaborativo, con ausencia de libro de texto y utilización del portafolio como material del alumno aumenta el rendimiento académico de los alumnos.

Se plantea una investigación longitudinal con un diseño de carácter cuasiexperimental de dos grupos, a partir de grupos naturales (la clase o aula).

El grupo A es el grupo de control, constituido por los alumnos que cursaron la materia el año anterior. El grupo experimental es el grupo B, alumnos que estudian la materia en el año de la investigación. En principio, los suponemos equivalentes por lo que no hacemos pretest.

El portafolio que diseñan los alumnos se realiza en papel, aunque se dio la opción de trabajarlo de manera digital, pero esta opción fue elegida por un número poco significativo de alumnos (tres en concreto). 


\section{Variables}

La variable independiente que se establece para el presente estudio es:

- La metodología consistente en la utilización del portafolio sin la existencia de libro de texto. Esta puntualización es importante, puesto que en todos los grupos de clase donde se ha llevado a cabo la experiencia se ha decidido optar por este sistema.

Respecto del curso anterior y en relación con las posibles variables extrañas, hay que decir que el uso del portafolio y la ausencia de libro de texto han llevado implícitos algunos cambios metodológicos, que no hemos podido aislar como variables.

a) Los complementos que hemos creído necesarios para guiar el proceso de elaboración del portafolio y que están dentro de la competencia de aprender a aprender, tales como el uso de la rúbrica de autoevaluación o aquellas estrategias dirigidas a enseñar al alumno a reflexionar y valorar su propio aprendizaje.

b) La explicación de la unidad, que no se realiza de forma magistral ni a partir de un texto, sino buscando sistemas más participativos, a través de fichas de trabajo, presentaciones por equipos, vídeos etc.

c) En cada unidad didáctica se exponen los objetivos de dicha unidad y el estudiante elabora una reflexión acerca de los objetivos (si le son familiares o no, si considera que va a tener especial dificultad en alguno de los aspectos etc.)

d) Después de cada unidad se realiza una autoevaluación (a veces coevaluación) para ver en qué medida se han logrado los objetivos.

La variables intervinientes necesitan ser controladas en la medida de lo posible mediante ciertas técnicas de control, proceso que entraña grandes dificultades comparándolo con el proceso de control en las ciencias experimentales (García Llamas, J.L. 2001). Una de las técnicas de mayor empleo en investigación educativa es la de mantener constantes los valores de la supuesta variable interviniente. Por ello tanto en el grupo de control como en este se han mantenido constantes los valores de dichas variables intervinientes, a saber:

a) Uso de TIC para realizar trabajos.

b) Utilización de trabajos colaborativos.

c) Enfoque práctico de la asignatura (audiciones, práctica instrumental etc.) 
La eliminación del libro de texto y el uso del portafolio en la asignatura de música en ESO: una experiencia para incrementar la calidad del aprendizaje y la motivación del alumnado

Katia Sanz Chouquet

Un estudio factorial de varios grupos con diversa combinación de variables puede darnos en un posterior estudio una medida más precisa de los efectos de las mismas.

Las variables dependientes que queremos estudiar son:

1. Interés y satisfacción del alumno con la asignatura

2. Rendimiento académico

Dado que estos conceptos son extremadamente amplios y en algunos aspectos difícilmente medibles, nuestros instrumentos de recogida de datos se orientan en diversas direcciones:

a) Por un lado los relativos a resultados académicos: la nota media en la asignatura al final de curso.

b) Por otro lado los relativos a opiniones (reflejados en el cuestionario y clima de aula).

\section{Instrumentos de recogida de datos}

\section{CUESTIONARIO DE CLIMA DE APRENDIZAJE}

Los planes de evaluación de sistemas han prestado mucha atención a la evaluación de variables de producto. Consideramos importante para conocer más en profundidad la situación del centro aplicar un cuestionario referido al contexto. Esto nos otorga un marco en el que analizar los resultados del resto de instrumentos, además de una orientación para mejorar los aspectos que aparezcan pobremente valorados. Nos ha parecido oportuno adaptar a nuestro alumnado el micro-instrumento propuesto por López-González, Tourón, Tejedor (2012) para medir el clima de aprendizaje. Este cuestionario tiene la ventaja de ser breve y claro, reuniendo los criterios de eficiencia, funcionalidad y eficacia. Está validado, como podemos ver en los procedimientos especificados en la López-González et al. (2012).

Contempla las siguientes dimensiones:

- Expectativas

- Satisfacción

- Cooperación

- Ambiente físico

- Competitividad

- Interés y preocupación 
La eliminación del libro de texto y el uso del portafolio en la asignatura de música en ESO: una experiencia para incrementar la calidad del aprendizaje y la motivación del alumnado

CALIFICACIONES FINALES DE LOS CURSOS ESTUDIADOS.

Se utilizan las calificaciones obtenidas tras realizar la media de las tres evaluaciones anuales. En cada una de estas evaluaciones se han tenido en cuenta las pruebas objetivas (exámenes), los trabajos colaborativos y proyectos varios, la actitud del alumno (puntualidad, interés, atención en clase, entrega de tareas en los plazos indicados), así como las valoraciones parciales de las actividades incluidas en el portafolio.

\section{CUESTIONARIO DE SATISFACCIÓN HACIA LA ASIGNATURA}

A la hora de confeccionar el cuestionario se procuró que la extensión fuera adecuada y los ítems estuvieran formulados con suficiente claridad. Se llegó a la versión definitiva tras un análisis de fiabilidad y una prueba piloto del mismo. Se elaboraron atendiendo a cuatro aspectos o dimensiones valorados mediante una escala Likert:

1. Interés

2. Objetivos y logros

3. Recursos

\section{Resultados}

\section{Datos cuantitativos: Descriptivos}

Los datos descriptivos se han centrado en la información obtenida de los alumnos del presente curso, es decir, aquellos que han recibido la nueva metodología sin libro de texto y con uso de portafolio.

\section{Cuestionario CLIMA DE APRENDIZAJE}

Del análisis de las frecuencias del cuestionario a través del programa informático se desprenden las siguientes conclusiones:

Los ítems relacionados con expectativas se encaminan a valorar la satisfacción con el aprendizaje en clase, al buen nivel al acabar el curso y la utilidad de lo aprendido de cara a buscar trabajo al final de ESO. La respuesta más repetida es "a veces", con $67.6 \%$, 57.7\% y $40.5 \%$ de elecciones. Es la respuesta más cómoda para el alumno y la que menos le implica. Merece la pena señalar sin embargo que hay cierta expectativa entre el alumnado de adquirir un buen nivel educativo y que el aspecto 
La eliminación del libro de texto y el uso del portafolio en la asignatura de música en ESO: una experiencia para incrementar la calidad del aprendizaje y la motivación del alumnado

Katia Sanz Chouquet

de "utilidad" sale mal parado, con un 30\% de elecciones de la respuesta "nunca". Es notoriamente alto este porcentaje, explicable en parte si pensamos que la muestra de alumnos tiene unas características sociales entre las que se incluyen hacer estudios universitarios. Ninguno contempla la posibilidad de abandonar los estudios al acabar Secundaria ni la posibilidad de trabajar para contribuir al gasto familiar.

En los ítems relacionados con la satisfacción, un $46.8 \%$ se siente bastante orgulloso de su trabajo en clase y un $44 \%$ de alumnos creen que los profesores siempre están contentos cuando ven que los alumnos aprenden y trabajan bien.

Los ítems relacionados con la cooperación en el aula valoran negativamente el hecho de que los alumnos dejen trabajar al resto. Los datos se agrupan sobre todo en los dos primeros valores (nunca, a veces), con un $74.8 \%$ de elecciones.

Sobre el ambiente de clase, se valora la distribución del aula con vistas al trabajo. De nuevo nos encontramos con valores mayoritarios en "a veces" (un $60.4 \%$ y un $53.1 \%$ respectivamente), aunque destaca la puntuación que la distribución de clase obtiene, con un 17\% de alumnos que piensa que dicha distribución no favorece en ningún caso el trabajo.

Respecto a la competitividad, solo un $9 \%$ de alumnos piensan que "siempre" está presente en los trabajos, mientras que el $45 \%$ se decanta por "a veces". En cuanto al interés, que se centra en la intención de aprender de los alumnos, destacar que nada menos que un 55\% por ciento elige "a veces", mientras que "siempre" obtiene la mínima puntuación $(8.1 \%)$.

\section{CUESTIONARIO DE SATISFACCIÓN E INTERÉS POR LA ASIGNATURA}

En primer lugar presentaremos los datos relativos a frecuencias de los ítems.

Ítems referentes a intereses

En este apartado se recoge el interés hacia diferentes aspectos de la música relacionados o no con el ámbito académico. Podemos considerar que el interés por la música en general es medio - alto, sin embargo desciende considerablemente cuando nos referimos a la historia de la música. En nuestra opinión, los alumnos identifican habitualmente "historia de la música" con "música clásica", mientras que el interés hacia 
la música en general es mayor, por estar relacionado con la escucha de la música como forma de ocio. En cuanto al grado de interés por la asignatura, los valores son más favorables que en los otros aspectos, lo cual es un dato llamativo en pro de la atención y motivación del estudiante. El interés por acercarse a otras músicas queda reflejado también y sus valores se distribuyen de manera que ninguno sobresale especialmente por encima de los demás. Presentamos un gráfico (figura 1) donde se muestra la puntuación total de cada aspecto (frecuencia x puntuación). En dicho gráfico observamos el resultado total de las valoraciones de cada ítem, donde sobresale la práctica musical. Para los alumnos la música se centra en la cuestión de tocar un instrumento, valorando menos el conocimiento teórico acerca de la misma o la reflexión.

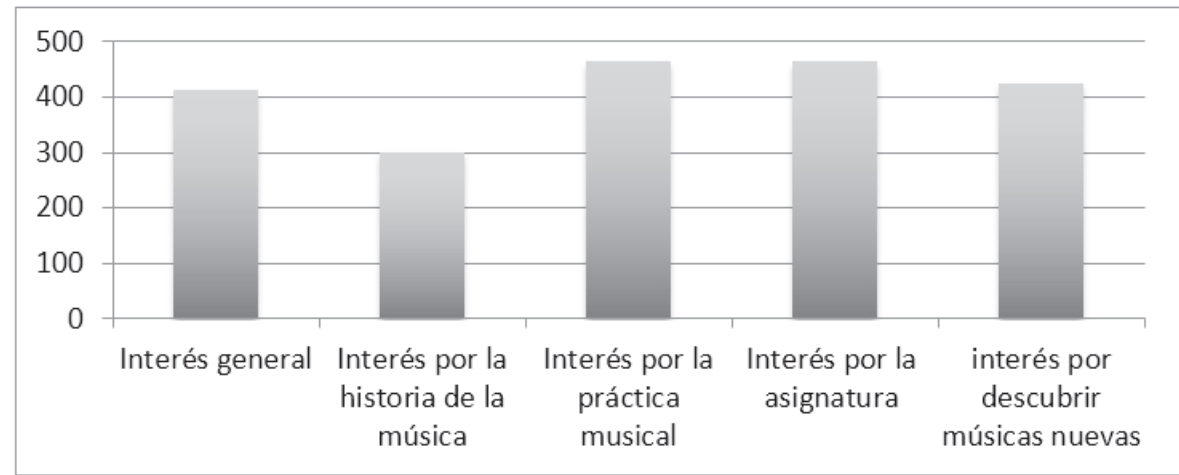

Figura 1. Interés mostrado por el alumno en los diferentes aspectos (puntuación total).

Ítems referentes a objetivos

La valoración de los objetivos de los alumnos para el curso (ver figura 2) se centra en aspectos como son la mejora de la práctica instrumental, de los conocimientos sobre compositores y músicos, de la capacidad auditiva, de la capacidad de apreciar estilos nuevos y de alcanzar las calificaciones más altas. 
La eliminación del libro de texto y el uso del portafolio en la asignatura de música en ESO: una experiencia para incrementar la calidad del aprendizaje y la motivación del alumnado

Katia Sanz Chouquet

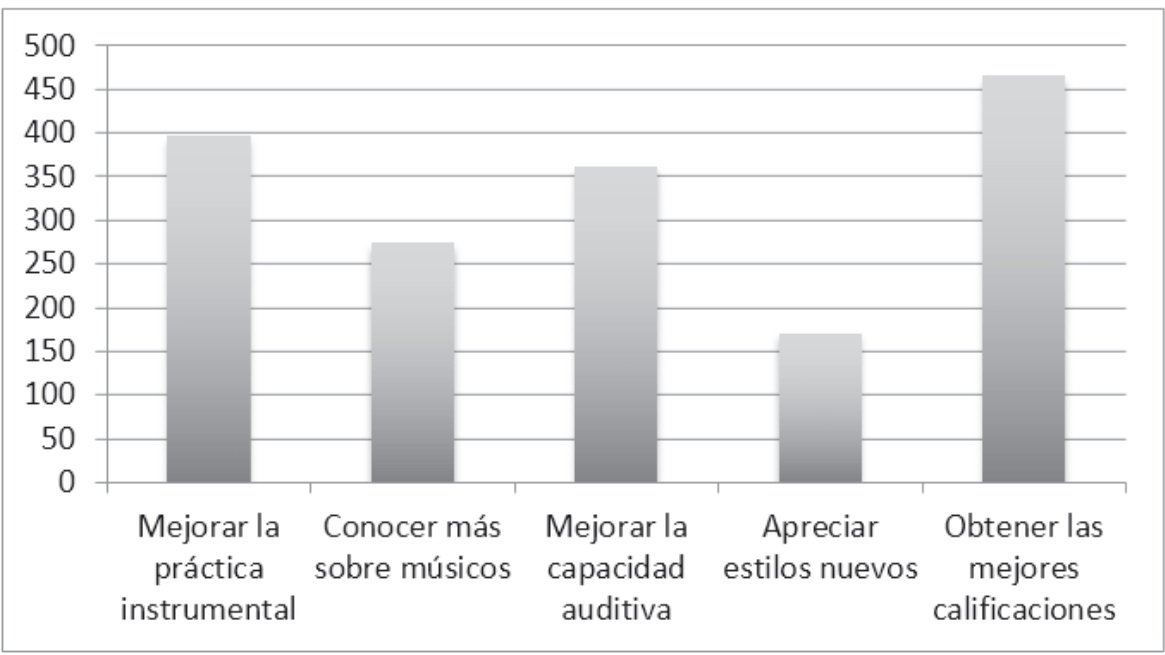

Figura 2. Objetivos que se marca el alumno de cara al curso escolar (puntuación total).

A pesar de que el grado de interés por la música no es especialmente alto, en cuanto a objetivos hay valoraciones medias-altas. Respecto al logro de objetivos, el que destaca por encima de todos es el de "obtener las mejores calificaciones", puntuado con la máxima valoración por un $48 \%$ del alumnado. Destaca en este sentido, aunque en menor medida, el objetivo de "mejorar la práctica instrumental" (un 51\% del alumnado elige los dos valores más altos de la escala) y "apreciar estilos nuevos" con un resultado similar al ítem anterior.

Respecto a los recursos que el alumno cree que mejoran su aprendizaje de manera notable, vemos en la figura 3 que, en general, todos se agrupan entre el 3 y el 4 respecto a la media de puntuaciones, salvo la elaboración de un cuaderno y el libro de texto (que es el recurso peor valorado). Destaca el uso del proyector e internet y el visionado de películas, como recursos favoritos para aprender. Todos ellos son recursos que el alumno ha manejado en un momento u otro de su vida académica. 
La eliminación del libro de texto y el uso del portafolio en la asignatura de música en ESO: una experiencia para incrementar la calidad del aprendizaje y la motivación del alumnado

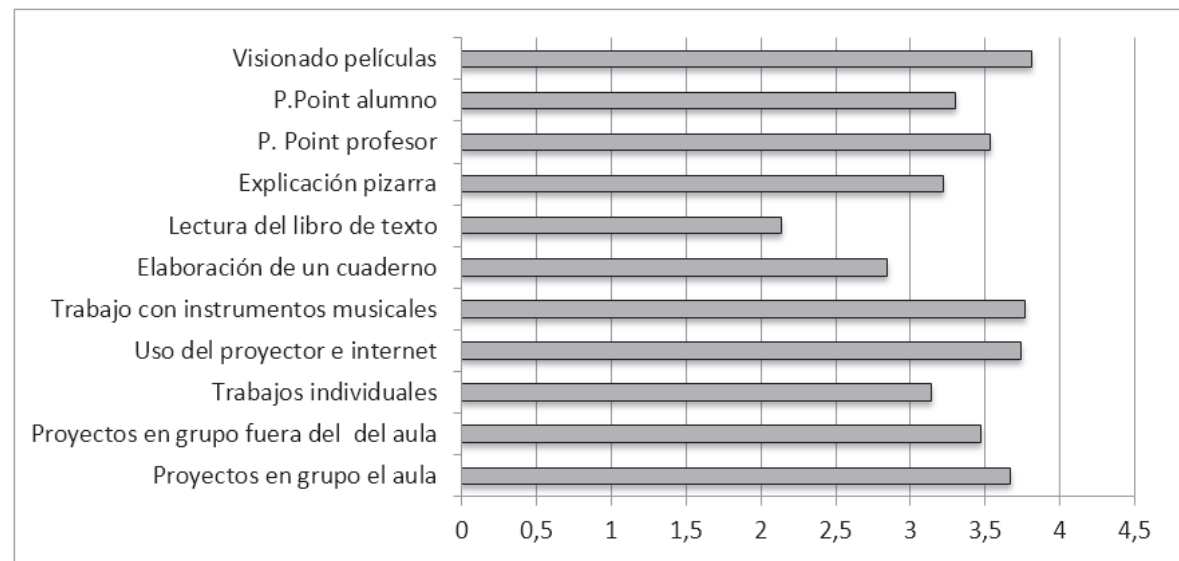

Figura 3. Recursos de aprendizaje preferidos por el alumno. (Media de las puntuaciones).

En cuanto a las capacidades que el alumno considera que ha mejorado este año a través de la asignatura, destacan la capacidad de trabajo en equipo y la capacidad de elección, cuyas medias superan en ambos casos el 3.5 (Ver figura 4).

Son relativamente pocos los alumnos que asignan puntuaciones bajas a su aprendizaje, si bien podemos destacar en contra de esta tendencia los resultados del ítem "manejo de herramientas informáticas", donde un $28 \%$ del alumnado valora en poca medida su aprendizaje.

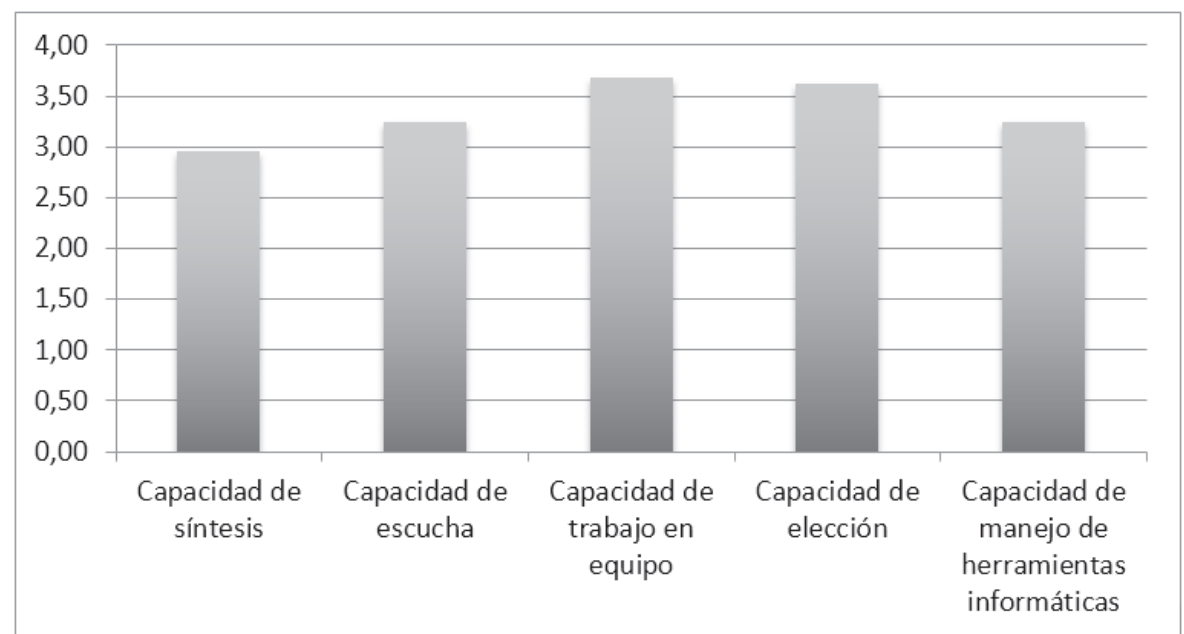

Figura 4. Capacidades que se han visto incrementadas según el alumno (media de puntuaciones). 
La eliminación del libro de texto y el uso del portafolio en la asignatura de música en ESO: una experiencia para incrementar la calidad del aprendizaje y la motivación del alumnado

Katia Sanz Chouquet

\section{Datos cuantitativos: Contraste}

A continuación relacionaremos los datos obtenidos entre los alumnos del grupo de control y el experimental en el cuestionario y en el rendimiento académico.

\section{Cuestionario}

En la comparativa de medias entre los resultados de ambos grupos, se muestra significatividad:

- En cuanto a intereses, es significativamente mayor el grado de interés por la historia de la música en el grupo experimental respecto al de control.

- En cuanto a objetivos, en el grupo experimental es significativamente mayor el de apreciar estilos nuevos respecto al grupo de control.

- En cuanto a recursos, es significativamente mayor en el grupo experimental el gusto por trabajar en proyectos en grupo fuera del aula y por la elaboración de un cuaderno respecto al grupo de control.

Para realizar el contraste entre los grupos de control y experimental se utilizó una prueba no paramétrica, dado que los datos del cuestionario están en nivel de medida ordinal. Resulta adecuada la prueba $U$ de Mann-Whitney como alternativa a la prueba $t$, por la característica del nivel de medida ya apuntada. Además es apta para utilizarla con tamaños muestrales desiguales como es nuestro caso. La prueba $U$ nos muestra con un intervalo de confianza del $95 \%$ que se da una mejoría significativa en el grupo experimental en al menos uno de los ítems asociados a cada dimensión del cuestionario:

- En cuanto a interés, ha aumentado el grado de interés por la historia de la música.

Tabla 1. Comparación de medias. Grado de interés por la historia de la músi-

ca.

\begin{tabular}{lll}
\hline \multirow{2}{*}{ Control } & Media & 2.08 \\
\cline { 2 - 3 } & Desviación estándar & .988 \\
\hline \multirow{2}{*}{ Experimental } & Media & 2.48 \\
\cline { 2 - 3 } & Desviación estándar & 1.198 \\
\hline
\end{tabular}


La eliminación del libro de texto y el uso del portafolio en la asignatura de música en ESO: una experiencia para incrementar la calidad del aprendizaje y la motivación del alumnado

- En cuanto a objetivos, es mejor valorado "apreciar estilos nuevos".

Tabla 2. Comparación de medias. Objetivo "apreciar estilos nuevos".

\begin{tabular}{cccc}
\hline & Control & \multicolumn{2}{c}{ Experimental } \\
\hline Media & Desviación estándar & Media & Desviación estándar \\
\hline 2.92 & 1.208 & 3.43 & 1.250 \\
\hline
\end{tabular}

- En cuanto a recursos, el cuaderno, a pesar de no ser muy valorado, es más apreciado en grupo experimental, así como las explicaciones en pizarra y PowerPoint y la elaboración de proyectos, como muestra la tabla 3.

Tabla 3. Comparación de medias. Recursos preferidos por el alumno para su aprendizaje.

\begin{tabular}{lcccc}
\cline { 2 - 5 } & \multicolumn{2}{c}{ Grupo de control } & Grupo experimental \\
\cline { 2 - 5 } & Media & $\begin{array}{c}\text { Desviación } \\
\text { estándar }\end{array}$ & Media & $\begin{array}{c}\text { Desviación } \\
\text { estándar }\end{array}$ \\
\hline $\begin{array}{l}\text { Proyectos en grupo realizados } \\
\text { fuera del aula }\end{array}$ & 3.00 & 1.326 & 3.47 & 1.183 \\
Elaboración de un cuaderno & 2.29 & 1.271 & 2.84 & 1.289 \\
$\begin{array}{l}\text { Explicación del profesor en la } \\
\text { pizarra }\end{array}$ & 2.69 & 1.087 & 3.23 & 1.087 \\
Explicación PowerPoint profesor & 3.05 & 1.265 & 3.53 & 1.059 \\
\hline
\end{tabular}

- En cuanto a capacidades que han mejorado según la opinión del alumno, resultan significativas la capacidad de síntesis y la de trabajo en equipo (ver tabla 4). Esta es especialmente interesante, puesto que aunque es una estrategia que se ha utilizado con parecida frecuencia en el grupo experimental y en el de control, en el primero se ha insistido mucho en pautas organizativas a partir de plantillas y dinámicas que los alumnos han incorporado a su portafolio.

Tabla 4. Comparación de medias. Capacidades que el alumno considera incrementadas en el curso académico.

\begin{tabular}{llcc}
\cline { 3 - 4 } & $\begin{array}{c}\text { Capacidad de } \\
\text { síntesis }\end{array}$ & $\begin{array}{c}\text { Capacidad de } \\
\text { trabajo en equipo }\end{array}$ \\
\hline \multirow{2}{*}{ Control } & Media & 2.59 & 3.23 \\
\cline { 2 - 4 } & Desviación estándar & 1.044 & 1.150 \\
\hline \multirow{2}{*}{ Experimental } & Media & 2.95 & 3.68 \\
\cline { 2 - 4 } & Desviación estándar & .985 & 1.052 \\
\hline
\end{tabular}


La eliminación del libro de texto y el uso del portafolio en la asignatura de música en ESO: una experiencia para incrementar la calidad del aprendizaje y la motivación del alumnado

Katia Sanz Chouquet

\section{RENDIMIENTO ACADÉMICO}

Se comparan las notas en la materia "música" entre los alumnos del grupo de control y los del grupo experimental. No se dan diferencias significativas a favor de nuestras hipótesis, aunque los datos deben ser leídos a la luz del resto de informaciones acerca de la experiencia, en aras de la complementariedad metodológica. Los cuestionarios arrojan resultados favorables a la incorporación de la metodología basada en el portafolio y sin libro de texto y valoramos muy positivamente esta experiencia.

\section{Discusión y conclusiones}

Nuestros objetivos iniciales acerca de la mejora de los procesos de aprendizaje e implicación del alumnado se han visto ampliamente cumplidos tras la experiencia llevada a cabo. Hemos podido constatar el incremento de interés y motivación por la materia. Esto ha sido posible gracias a la reflexión sobre dichos procesos y al uso de diferentes recursos metodológicos más allá del libro de texto. Los alumnos se decantan principalmente por los trabajos por proyectos como recurso predilecto, además de la elaboración del "cuaderno", denominación generalizada por ellos para el portafolio o carpeta de aprendizaje. También hemos podido confirmar que, según otra de nuestras hipótesis, el uso del portafolio potencia en el alumno la capacidad de síntesis y la del trabajo en equipo, según la metodología específica de trabajo que se utilizó en la experiencia. En la misma línea de la mejora en el trabajo colaborativo y en capacidades de organización y síntesis están otras investigaciones anteriores en el ámbito del portafolio (Gallardo \&Sierra, 2015, Villalustre \& Moral, 2010). En cuanto al manejo de las TIC, este no se ven especialmente incrementado. Hay que señalar que, en palabras de muchos de los alumnos al rellenar el cuestionario, esto sucedía porque ya consideraban que manejaban estas herramientas suficientemente bien antes de comenzar el curso, por lo cual el incremento no ha sido reflejado de manera tan evidente como en los otros aspectos estudiados en lo que a capacidades se refiere. El hecho de haber optado por portafolios convencionales en lugar de electrónicos también condiciona este resultado.

Los datos cuantitativos en cuanto al rendimiento del alumno no han evidenciado diferencias significativas respecto al grupo de control.

Sin embargo los cuestionarios de satisfacción con la asignatura nos 
muestran un panorama muy favorable al uso del portafolio. Dado que la constatación de esta mejora se basa en autoinformes de los propios alumnos, lógicamente debemos tomar los datos con la cautela que este tipo de instrumentos implican: los alumnos opinan que sí han mejorado en algunas capacidades, además de haberse incrementado su interés por la historia de la música y la apreciación de nuevos estilos. Lo que no podemos negar es que la metodología utilizada ha fomentado el interés de los alumnos, lo cual es muy positivo.

El uso del portafolio implica un cambio metodológico que, entre otras cosas, aumenta la responsabilidad del estudiante, pero este uso debe ir acompañado del desarrollo de instrumentos amplios de evaluación (Arceo et al., 2011). Nos encontramos ante la difícil tarea de integrar datos de diversa índole para definir o describir un panorama creado tras la implantación del uso del portafolio y el cambio metodológico que ha traído consigo. Creemos que si se dan las instrucciones precisas al alumnado se pueden obtener muy buenos resultados. Para ello es necesaria una evaluación formativa con la suficiente periodicidad como para que el alumno incorpore mejoras muy frecuentemente. Conviene tener en cuenta que el portafolio mejora los procesos de evaluación del aprendizaje (Fimia \& Moreno, 2012) y nos conduce a una evaluación para el aprendizaje (Hernández, 2008).

Nuestra intención se encamina a continuar con esta línea de trabajo en los años posteriores. Creemos que al alumno le tomará un tiempo acostumbrarse a la dinámica de trabajo que proponemos y por ello sería positivo retomar el portafolio en el curso siguiente, de manera que se asentara dicha práctica como habitual. Consideramos que la investigación sería más completa incorporando una perspectiva cualitativa: entrevistas y análisis de contenido de los portafolios, además de un diario del docente.

No hemos podido aislar qué parte de los resultados positivos se deben al hecho de realizar un portafolio y cuáles a las estrategias que hemos utilizado para autoevaluación, planificación y reflexión, pero creemos que todo forma parte de un mismo enfoque: concienciar al alumno como protagonista de su aprendizaje. Probablemente esa es la clave para incentivar el interés y la motivación del alumnado. Sería muy recomendable poder proseguir esta línea de investigación clarificando la influencia de estas variables. 
La eliminación del libro de texto y el uso del portafolio en la asignatura de música en ESO: una experiencia para incrementar la calidad del aprendizaje y la motivación del alumnado

Katia Sanz Chouquet

\section{Referencias bibliográficas}

Alsina, M., \& Godoy, J. (2009). Competencias creativas en el aula de música. Eufonía, Didáctica de la Música, 45, $97-107$.

Alsina, P. (2008). La evaluación por portafolios en el aula de música. En A. Giráldez (ed.), Percepción y expresión en la cultura musical básica (pp.45-60). Madrid: Ministerio de Educación, cultura y deporte.

Arceo, F. D. B., Martínez, E. R., \& Sánchez, A. H. (2011). La carpeta electrònica com a instrument per a la reflexió sobre el desenvolupament professional i la formació en estudiants de postgrau. Observar, (5), 7-20.

Barberà, E. (2005). La evaluación de competencias complejas: la práctica del portafolio. Educere, vol. 9 (31) octubre-diciembre, 487-503.

Barberà, E. et al. (2009). Portafolios electrónicos y educación superior en España. Situación y tendencias. Red U - Revista de Docencia Universitaria. Número monográfico III. Portafolios electrónicos y educación superior en España (en coedición con RED) 7 de Abril de 2009. Consultado el 20/08/2016 en http://www.um.es/ead/Red_U/m3/

Barragán Sánchez, R. (2005). El portafolio: Metodología de evaluación y aprendizaje de cara al nuevo espacio europeo de educación superior. Una experiencia práctica en la universidad de Sevilla. Revista Latinoamericana De Tecnología Educativa, 4(1), 121-139.

Barrett, H. (2005). White paper: Researching electronic portfolios and learner engagement. The reflect initiative. Consultado el 20/08/2016 de:

http://electronicportfolios.org/reflect/whitepater.pdf

Calfee, R. C., \& Perfumo, P. (1993). Carpetas de estudiante: Oportunidades para una revolución en la evaluación. Comunicación, Lenguaje y Educación, 5(19-20), 87-97. doi:10.1080/02147033.1993.10821090

Corominas Rovira, E. (2000). ¿Entramos en la era portafolio? Bordón: Revista de Orientación Pedagógica, 52(4), 509-521.

Crawford, R. (2014). A multidimensional/non-linear teaching and learning model: Teaching and learning music in an authentic and holistic context. Music Education Research, 16, 50 -69. doi:10.1080/14613808.2013.812627

Estebaranz García, A. y Mingorance Díaz, P. (1995). ¿Cómo mejorar el impacto de la evaluación sobre los procesos de enseñanza-aprendizaje en la educación secundaria obligatoria? Enseñanza \& Teaching: Revista interuniversitaria de didáctica, (13), 223240.

Fimia, Y. \& Moreno, I. (2012). El portafolio digital y su impacto en la calidad del proceso de evaluación del aprendizaje. EDUTEC, Revista Electrónica de Tecnología Educativa, 39. Consultado el 20/08/2016 de:

http://edutec.rediris.es/Revelec2/Revelec39/portafolio_digital_impacto_calidad_ proceso_e valuacion_aprendizaje.html

Gallardo Gil, M., \& Sierra Nieto, E. (2015). El Portafolios de los estudiantes como estrategia alternativa a las pruebas estandarizadas para la evaluación de competencias. Qualitative Research in Education, 4(1), 72-102. doi: 10.4471/qre.2015.57

Gallego, D., Cacheiro, M. L., Martín, A. M., \& Angel, W. (2009). El e-portfolio como 
La eliminación del libro de texto y el uso del portafolio en la asignatura de música en ESO: una experiencia para incrementar la calidad del aprendizaje y la motivación del

alumnado

Katia Sanz Chouquet

estrategia de enseñanza y aprendizaje. Edutec. Revista Electrónica De Tecnología Educativa, (30) DOI: http://dx.doi.org/10.21556/edutec.2009.30.444

García Llamas, J.L. et al. (2001) Introducción a la investigación en educación. Madrid: Universidad Nacional de Educación a Distancia

Gregori, E., Bautista, G., Espasa, A., \& Guasch, T. (2006). Portfolio electrónico: DesarroIlo de competencias profesionales en la red. The Electronic Portfolio: Development of Professional Competencies using the Web, 3(2)

Hernández, F. H. (2008). El portafolio electrónico y el aprendizaje para la comprensión en secundaria. Investigación en la Escuela, (64), 59-72.

Klenowski, V. (2005). Desarrollo de portafolios para el aprendizaje y la evaluación: procesos y principios (Vol. 98). Madrid: Narcea

López, J. L. (2010). Un instrumento para múltiples usos: la carpeta o portfolio. Revista de ciencias de la educación: Órgano del Instituto Calasanz de Ciencias de la Educación, (222), 241-254.

López González, E., Tourón Figueroa, J., \& Tejedor Tejedor, F. J. (2012). Diseño de un micro-instrumento para medir el clima de aprendizaje en cuestionarios de contexto. Bordón. Revista De Pedagogía, 64(2), 111-126. Adaptación de Katia Sanz Chouquet.

Lorenzo-Quiles, O., Vílchez-Fernández, N., \& Herrera-Torres, L. (2015). Educational effectiveness analysis of the use of digital music learning objects. Comparison of digital versus non-digital teaching resources in compulsory secondary education. Infancia y Aprendizaje, 38(2), 295-326. DOI: 10.1080/02103702.2015.1016748

Martínez Lirola, M. (2008). El uso del portfolio como herramienta metodológica y evaluadora en el proceso de convergencia europea. Profesorado: Revista De Curriculum y Formación Del Profesorado, 12(2) 1-12

Medina Rivilla, A., \& Domínguez Garrido, M. C. (2009). Didáctica: Formación básica para profesionales de la educación. Madrid: Universitas.

Medina Rivilla, A., \& Salvador Mata, F. (2009). Didáctica general (2 ${ }^{\text {a }}$ ed.). Madrid: Prentice Hall.

Monereo, C. (2010). ¡Saquen el libro de texto!: resistencia, obstáculos y alternativas en la formación de los docentes para el cambio educativo. Revista de educación, (352), 583-597.

Juste, R. P. (2000). La evaluación de programas educativos: conceptos básicos, planteamientos generales y problemática. Revista de investigación educativa, 18(2), 261-287.

Pérez Juste, R. coord. (2012.). El portfolio. Aprendizaje, competencias y evaluación. Madrid: UNED.

Quintana, H. (1996). El portafolio como estrategia para la evaluación. Teoría y Didáctica de la Lengua y la Literatura, 8, 89-96.

Rhodes, T. L. (2010). Making learning visible and meaningful through electronic portfolios. Change: The Magazine of Higher Learning, 43 (1), 6-13.

Rodríguez Illera, J. L., Galván Fernández, C. y Martínez Olmo, F. (2013). El portafolios digital como herramienta para el desarrollo de competencias transversales en el alumnado. Revista Teoría de la Educación: Educación y Cultura en la Sociedad de la Información. 14(2), 157-177 Consultado el 20/08/2016 de:

http://campus.usal.es/ revistas_trabajo/index.php/revistatesi/article/view/10218/10627 
La eliminación del libro de texto y el uso del portafolio en la asignatura de música en ESO: una experiencia para incrementar la calidad del aprendizaje y la motivación del alumnado

Katia Sanz Chouquet

Rodríguez, V. S. (2011). Innovaciones metodológicas en Educación Secundaria: TIC, música y medios audiovisuales. Edetania: estudios y propuestas socio-educativas, (39), 151-157.

Sánchez, J. E. (2011). Los fines de la Educación Secundaria en la sociedad española actual. Edetania: estudios y propuestas socio-educativas, (39), 43-56.

Sevillano García, M. L., Bartolomé Crespo, D., \& Pascual Sevillano, M. Á. (2007). Investigar para innovar en enseñanza. Madrid: Pearson Educación.

Shackelford, R. L. (1996). Student Portfolios: A Process/Product Learning and Assessment Strategy. Technology Teacher, 55(8), 31-36.

Villalustre Martínez, L., \& Moral Pérez, M. E. D. (2010). E-portafolios y rúbricas de evaluación en ruralnet. Pixel-Bit. Revista de Medios y Educación, 37, 93-105

Wolf, D. P. (1989). Portfolio assessment: Sampling student work. Washington, D.C: Association for Supervision and Curriculum Development. 